\title{
Sustitución de nitrito de sodio por antocianinas de flores de mastuerzo atomizado, en el color, capacidad antioxidante y aceptabilidad de salchichas tipo Frankfurt
}

\section{Substitucion of sodium nitrite by anthocyanins of atomized mastuerzo flowers, incolor, antioxidant capacity and accetability of Frankfurt type sausages}

\author{
Espinoza Silva, Clara Raquel; Quispe Solano, Miguel Ángel \\ Facultad de Ingeniería en Industrias Alimentarias, Universidad Nacional del Centro del Perú \\ Email: crespinoza@uncp.edu.pe
}

\section{Resumen}

La presente investigación, tiene como objetivo sustituir el nitrito de sodio por el extracto antociánico atomizado de los pétalos anaranjados de flores de mastuerzo (Tropaeolum majus L.) en salchichas tipo Frankfurt. El estudio se encuentra dentro del tipo de investigación aplicada y nivel experimental, para lo cual se realizó la extracción del pigmento antocianico del mastuerzo encapsulándose con maltodextrina $(10 \mathrm{DE})$ a $9 \%$ y secada por atomización en un mini spray dryer B-290 a $110{ }^{\circ} \mathrm{C}$ dando 1100,43 mg pelargonidina-3-glu/100g de antocianinas y 1519,84 $\mu \mathrm{mol}$ Trolox/g de capacidad antioxidante. El nitrito de sodio se sustituyó por el atomizado de antocianinas en la formulación de salchichas tipo Frankfurt en proporciones de $50 \%, 75 \%$ y $100 \%$. Después del escaldado de las salchichas se evidenció la presencia de antocianinas entre 0,09 a $0,55 \mathrm{mg} / 100 \mathrm{~g}$ de antocianinas y 9,25 a 20,30 $\mu \mathrm{mol} / \mathrm{g}$ de capacidad antioxidante entre las diferentes sustituciones $(\mathrm{p}<0,05)$. También, se encontró que la formulación que presentó los mejores atributos en color $(\mathrm{L} *=63,31 ; \mathrm{a} *=5,99, \mathrm{~b} *=13,45 \mathrm{y}$ Rango 58,81) y sabor (Rango 57) fue la sustitución al $50 \%$; no se evidenció diferencia estadística $(\mathrm{p}>0,05)$ con respecto al olor y textura, las salchichas, en las diferentes sustituciones, se encuentran dentro de los límites permitidos para la carga microbiana establecidos en la NTS-N ${ }^{\circ} 071$.

Palabras clave: Atomización, antocianina, mastuerzo, salchicha, nitratos.

\begin{abstract}
The objective of this research is to replace sodium nitrite by atomized anthocyanin extract of the orange petals of mastuerzo flowers (Tropaeolum majus L.) in Frankfurt type sausages, the study type is applied research and experimental level, to that purpose anthocyanin pigment of the mastuerzo was extracted by encapsulating with maltodextrin (10 DE) at $9 \%$ and dried by atomization in a Mini spray dryer B-290 at $110{ }^{\circ} \mathrm{C}$ giving $1100.43 \mathrm{mg}$ pelargonidin-3-glu/100 $\mathrm{g}$ of anthocyanins and $1519.84 \mu \mathrm{mol}$ Tro-lox/g of antioxidant capacity. Sodium nitrite was replaced by anthocyanin atomization in the formulation of Frankfurt-type sausage in $50 \%, 75 \%$ and $100 \%$ proportions. After sausage scalding, a presence of anthocyanins of 0.09 to $0.55 \mathrm{mg} / 100 \mathrm{~g}$ of anthocyanins and 9.25 to $20.30 \mu \mathrm{mol} / \mathrm{g}$ of antioxidant capacity was observed among the different substitutions $(p<0.05)$. It was also found that the formulation which presented the best attributes in color $(L *=63.31, a *=5.99$, $b^{*}=13.45$ and Rank 58.81) and flavor (Rank 57) was the $50 \%$ substitution; no statistical difference was observed $(p>0.05)$ with respect to smell and texture, sausages in the different substitutions are within permitted limits for the microbial load established in NTS-N ${ }^{\circ} 071$.
\end{abstract}

Keywords: Atomization, anthocyanin, cress, sausage, nitrates 


\section{Introducción}

Los aditivos sintéticos, advierten riesgo para la salud, y como consecuencia de ello existe una creciente búsqueda de fuentes naturales para su extracción. El mastuerzo (Tropaeolum majus L.), conocido como capuchina, es una planta silvestre que crece en Perú; presenta un alto contenido de compuestos fenólicos (antocianinas, flavonoides, taninos) con estructura química especialmente adecuada para ejercer una acción antioxidante, además de poseer un poder colorante natural.

Los colorantes naturales son considerados, en general, como inocuos y consecuentemente las limitaciones específicas en su utilización son menores que las que afectan a los colorantes artificiales; así mismo, gracias al color se perciben sensaciones agradables a la vista, además de ser un factor estético.

En el procesamiento de embutidos se utilizan nitritos y nitratos de sodio o de potasio, que son aditivos químicos que evitan el ataque de microorganismos descomponedores y mejoran el color del producto final. Sin embargo, el uso estos aditivos puede provocar toxicidad, el cual se manifiesta como metahemoglobinemia y formación de nitrosaminas que es asociado a riesgo de cáncer. (Rodas, 2005)

La investigación en la industria de alimentos se centra continuamente en la adaptación y el desarrollo de nuevas formulaciones con la finalidad de mejorar la calidad y sobre todo la seguridad de los productos alimenticios (Ligianne et al., 2011), los metabolitos secundarios de las plantas se utilizan para productos farmacéuticos, cosméticos y con fines nutricionales, así como aditivos naturales en alimentos (Viviane et al., 2015). Los mismos que pueden cambiar los atributos de calidad referidos al color, olor, sabor y textura.

Las flores se consideran un alimento funcional, al aportar compuestos fenólicos, especialmente las de coloración oscura, por presentar la más alta composición fenólica y capacidad antioxidante (Lara et al., 2014). Por lo que se debe establecer la proporción de colorante natural de los pétalos Tropaleum majus L. que mantiene estable y fija el color de embutidos.

Por lo tanto, el objeto de este estudio fue evaluar el efecto de la sustitución del nitrito de sodio por el extracto antociánico atomizado de los pétalos anaranjados de flores de mastuerzo (Tropaeolum majus L.) en las propiedades físicas (color), fisicoquímicas (antocianinas y capacidad antioxidante), sensoriales y microbiológicas de las salchichas tipo Frankfurt.

\section{Materiales y métodos}

Tipo de Investigación: Aplicada

Nivel de investigación: Experimental

Lugar de ejecución: Laboratorio de Ciencia y Tecnología de la FAIIA y Laboratorio del Centro Experimental El Mantaro.

Unidad muestral: Salchichas tipo Frankfurt (65-75 g/ cada unidad)
Materiales: Los pétalos de mastuerzo anaranjado (Tropaeolum majus $L$.) fueron sembrados y recolectados en la Estación Experimental El Mantaro (Jauja-Junín-Perú) de la Universidad Nacional del Centro del Perú.

\section{Métodos}

\section{Obtención del extracto atomizado:}

Se separó los pétalos de las flores de mastuerzo para ser secado a temperatura ambiente $\left(15-18{ }^{\circ} \mathrm{C}\right)$, las que se trituraron entre 2000 a $3000 \mathrm{rpm}$ por 4 minutos, el polvo se mezcló con solvente (etanol-agua al 1:1 acidificado al $0,03 \% \mathrm{p} / \mathrm{v}$ con ácido cítrico) en una proporción de 1:20 (harina/solvente) por 24 horas a $4{ }^{\circ} \mathrm{C}$, luego la mezcla fue centrifugado a $4000 \mathrm{rpm}$ por 15 minutos, filtrado y concentrado al vacío $\left(40^{\circ} \mathrm{C}, 300\right.$ mbar y $\left.50 \mathrm{rpm}\right)$, luego el concentrado se mezcló con PVPP (polivinilpolipirrolidona) hidratado $(0,5 \mathrm{~g} / \mathrm{L})$ siguiendo el método propuesto por Giusti y Wrolstad (2001), enseguida el purificado se mezcló con maltodextrina (10 DE) a una concentración de $9 \%$ las que finalmente se secaron por atomización a una temperatura de entrada de aire de $110{ }^{\circ} \mathrm{C}$.

\section{Obtención de las salchichas tipo Frankfurt}

Para la obtención de las salchichas tipo Frankfurt se utilizó la formulación propuesta por Solís (2005); todos los insumos se pesaron de acuerdo a la Tabla 1.

Tabla 1. Formulación de salchichas tipo Frankfurt

\begin{tabular}{|c|c|c|c|c|}
\hline \multirow{2}{*}{ Insumos } & \multirow{2}{*}{$\begin{array}{l}\text { Testigo } \\
\text { (g) }\end{array}$} & \multicolumn{3}{|c|}{ Sustitución (g) } \\
\hline & & $100 \%$ & $75 \%$ & $50 \%$ \\
\hline Carne de cerdo & 700,00 & 700,00 & 700,00 & 700,00 \\
\hline $\begin{array}{l}\text { Grasa dorsal de } \\
\text { cerdo }\end{array}$ & 300,00 & 300,00 & 300,00 & 300,00 \\
\hline $\begin{array}{l}\text { Sal de cura (con } \\
25 \% \text { de nitrito de } \\
\text { sodio) }\end{array}$ & 0,42 & 0,00 & 0,11 & 0,21 \\
\hline Cloruro de sodio & 30,00 & 30,00 & 30,00 & 30,00 \\
\hline Pimienta molida & 0,50 & 0,50 & 0,50 & 0,50 \\
\hline Comino molido & 1,00 & 1,00 & 1,00 & 1,00 \\
\hline Ajos & 4,00 & 4,00 & 4,00 & 4,00 \\
\hline Orégano en polvo & 0,50 & 0,50 & 0,50 & 0,50 \\
\hline Polifosfato & 2,00 & 2,00 & 2,00 & 2,00 \\
\hline Almidón & 110,00 & 110,00 & 110,00 & 110,00 \\
\hline Hielo & 200,00 & 200,00 & 200,00 & 200,00 \\
\hline Humo líquido & 0,68 & 0,68 & 0,68 & 0,68 \\
\hline Colorante carmín & 1,00 & 0,00 & 0,00 & 0,00 \\
\hline Extracto atomizado & 0,00 & 9,54 & 7,16 & 4,77 \\
\hline
\end{tabular}

Nota: La dosis máxima de nitratos según Codex Alimentarius es $15 \mathrm{mg} / 100 \mathrm{~g}$ de carne.

\section{Cuantificación de antocianinas monoméricas}

La cuantificación de antocianinas monoméricas se determinó por el método $\mathrm{pH}$ diferencial propuesto por Giusti y Wrolstad (2001) con algunas modificaciones, se calculó utilizando la siguiente formula: 
Antocianina monomérica $(\mathrm{mg}$ pgd-3-glu $/ \mathrm{L})=\frac{\mathrm{A} \times \mathrm{PM} \times \mathrm{FD} \times 1000}{\mathrm{a} /}$

Dónde: A es la absorbancia, PM es el peso molecular, FD es el factor de dilución, $\varepsilon$ es la absorbancia molar o coeficiente de extinción molar, $\ell$ es la longitud de recorrido en $\mathrm{cm}$.

\section{Cuantificación de la capacidad antioxidante por DPPH・ (TEAC)}

La determinación de capacidad antioxidante se realizó mediante el método DPPH• propuesto por Brand-Williams et al. (1995) con algunas modificaciones por Kim et al. (2002). Los resultados fueron calculados en porcentaje de inhibición y expresados mg de trolox equivalente TEAC/g de muestra (figura 1).

$$
\% I=\left(\frac{A_{0}-A_{m}}{A_{0}}\right) \times 100
$$

Donde:

$\mathrm{A}_{0}=$ Absorbancia del blanco de muestra $(2850 \mu \mathrm{L}$ de DPPH $\bullet$ $+150 \mu \mathrm{L}$ de metanol al $80 \%$ ), después de 20 minutos.

$\mathrm{A}_{\mathrm{m}}=$ Absorbancia de la muestra $(2850 \mu \mathrm{L}$ de DPPH $\bullet+$ $150 \mu \mathrm{L}$ de extracto de muestra), después de 20 minutos.

Figura 1. Curva de calibración de trolox equivalente

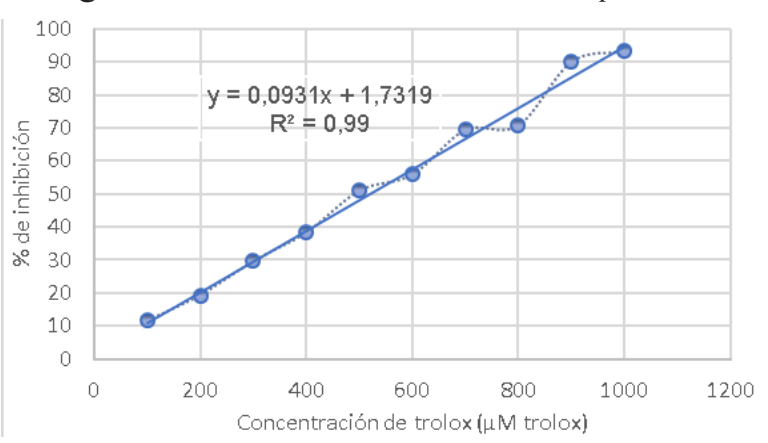

Evaluación de color en las salchichas tipo Frankfurt

Se determinó el color de las salchichas tipo Frankfurt antes y después de escaldado con el uso del colorímetro Konica Minolta CR-400 en el espacio de color Yxy e iluminante $\mathrm{C}$; Las mediciones de color se expresaron en luminosidad $\mathrm{L}^{*}$, y parámetros de cromaticidad $\mathrm{a}^{*} \mathrm{y} \mathrm{b}^{*}$.

\section{Evaluación sensorial de las salchichas tipo Frankfurt}

Para el análisis sensorial se realizó un análisis afectivo de aceptación, mediante una escala hedónica de 7 puntos ( 1 menor aceptación del atributo y 7 mayor aceptación del atributo) a través de una ficha de evaluación aplicadas a 32 panelistas (no entrenados) a los que se dieron las salchichas con las diferentes sustituciones evaluándose las variables de color, olor, sabor y textura según el método propuesto por Liria (2007).

\section{Evaluación microbiológica de las salchichas tipo Frankfurt}

Las muestras de salchichas se analizaron microbiológicamente en placa Petrifilm para recuento de aerobios mesófilos, E. coli, Staphylococcus aureus, Clostridium perfringens, salmonella sp y Listeria monocytogenes utilizando el método rápido, utilizando placas Petrifilm ${ }^{\mathrm{TM}} 3 \mathrm{M}^{\mathrm{TM}}$ (2009).

\section{Diseño experimental y análisis estadístico}

Para la evaluación de la sustitución de nitrito de sodio por el mejor extracto atomizado de antocianinas con respecto al contenido de antocianinas, capacidad antioxidante y color $\left(L^{*}, a^{*}\right.$ y $\left.b^{*}\right)$ se utilizó el diseño completamente aleatorio (DCA) con un nivel de significancia del $5 \%$, al encontrarse diferencias significativas se aplicó la prueba de comparación de medias deTukey, con respecto a la evaluación sensorial (COlor, olor, sabor y textura) se aplicó la prueba Kruskal Wallis a un nivel de significación de 5\% todos los datos fueron procesados en el programa estadístico SPSS statistics_Versión 22.

\section{Resultados y discusiones}

Antocianina y capacidad antioxidante (TEAC) de los pétalos anaranjados, extracto y atomizado del extracto de mastuerzo (Tropaeolum majus L.).

La Tabla 2, presenta la cuantificación de la antocianina y compuesto fenólico del pétalo de mastuerzo (Tropaeolum

Tabla 2. Contenido de antocianina monomérica y TEAC de los pétalos anaranjados, extracto y atomizado de antocianico de pétalos de mastuerzo (Tropaeolum majus L.).

\begin{tabular}{|c|c|c|c|c|c|c|c|c|c|}
\hline \multirow[t]{2}{*}{ Componentes } & \multicolumn{3}{|c|}{$\begin{array}{c}\text { Pétalos anaranjados } \\
\text { de mastuerzo }\end{array}$} & \multicolumn{3}{|c|}{$\begin{array}{l}\text { Extracto de antociani- } \\
\text { nas de mastuerzo }\end{array}$} & \multicolumn{3}{|c|}{$\begin{array}{c}\text { Atomizado de an- } \\
\text { tocianinas de mas } \\
\text { tuerzo }\end{array}$} \\
\hline & $\bar{x}$ & & $\sigma_{x}$ & $\bar{x}$ & & $\sigma_{x}$ & $\bar{x}$ & & $\sigma_{x}$ \\
\hline Antocianina monomérica (mg pgd-3-glu/100 g) & 94,82 & \pm & 0,01 & 186,94 & \pm & 1,99 & 1100,43 & \pm & 11,09 \\
\hline \% Acidez (Eq. ác. cítrico) & - & - & - & 0,35 & \pm & 0,01 & - & - & - \\
\hline${ }^{\circ}$ Brix a $20{ }^{\circ} \mathrm{C}$ & - & - & - & 8,75 & \pm & 0,25 & - & - & - \\
\hline $\mathrm{pH}$ a $20{ }^{\circ} \mathrm{C}$ & - & - & - & 4,78 & \pm & 0,01 & - & - & - \\
\hline Densidad aparente $(\mathrm{g} / \mathrm{mL})$ & - & - & - & 1,01 & \pm & 0,01 & - & - & - \\
\hline
\end{tabular}

Nota: El contenido de antocianinas esta expresada en pelargonidina-3-glucosido y el atomizado de antocianinas del mastuerzo se obtuvo con $9 \%$ de maltodextrina a temperaturas de entrada de aire de $110^{\circ} \mathrm{C}$, para $\mathrm{n}=3$. 
majus L.), el extracto antocianico y el atomizado de antocianinas según los protocolos indicados.

El contenido de antocianinas hallado en los pétalos frescos de mastuerzo anaranjado se aproxima a lo reportado por Garzón y Wrolstad (2009), quien encontró 72,00 $\mathrm{mg} / 100 \mathrm{~g}$, Souto et al. (2012), reporta 78,36 mg/100 $\mathrm{g}$, ambos autores expresaron en mg de cianidina-3-glucosido y que la variabilidad depende del lugar de procedencia y pueden ser afectados por factores ambientales como el clima, altitud, luz solar y la temperatura.

Este contenido superior en antocianinas es importante en diversas aplicaciones y usos en la industria alimentaria, ya que dicho contenido es comparable con las antocianinas de diversas matrices alimentarias como el arándano rojo con 34,00 mg/100 g (cy-3-glu) y la fresa con 71,80 mg/100 g (pgd-3-glu) (Moyer et al., 2002).

La actividad antioxidante, es la capacidad de los compuestos fenólicos como: flavonoides, antocianinas, carotenoides, taninos, ácidos fenólicos y otros (Badui, 2006), de reaccionar con especies reactivas de oxígeno (cualquier átomo o molécula con electrones desapareados que son capaces de atacar a proteínas, carbohidratos, grasas, ADN del cuerpo, ejerciendo un efecto oxidativo que daña las células) (Youngson, 2004). De esta manera, los pétalos anaranjados de mastuerzo, son una fuente rica en capacidad antioxidante por contener todas las sustancias antes mencionadas. Garzón y Wrolstad (2009), reportan contenido de 91,87 $\mu \mathrm{mol}$ trolox/g cercano a lo hallado en la investigación, ello indica que las flores son una fuente promisoria para su aprovechamiento.

Para la obtención del extracto antociánico, la temperatura de lixiviado es muy importante, debido que a temperaturas elevadas menores en los tiempos de extracción, se corre el riesgo de extraer solutos indeseables; en cambio, la extracción por frío requiere de periodos más largos (López, 2008), por ello se empleó una temperatura de 4 ${ }^{\circ} \mathrm{C}$ por 24 horas para proteger a los pigmentos antociánicos y evitar su degradación. Zapata (2014), utilizó la solución de extracción etanol-agua (1:1) acidificado con ácido cítrico al 1\% para la extracción de antocianinas a partir de arándanos, por el cual se apreció mayor contenido de antocianinas en esta combinación de solvente y estabilidad por parte de ácido cítrico empleado al 0,03\%.

De acuerdo a los valores obtenidos de antocianina monomerica en los extractos atomizados, estos se ven afectados por las diferentes temperaturas de atomización, encontrando la tendencia a menor temperatura de secado existe mayor contenido de antocianinas (Revelo, 2014), en cuanto a mayor concentración de maltodextrina hay mayor dispersión de pigmento. El mayor contenido de antocianina se encuentra con $9 \%$ de maltodextrina a una temperatura de salida de aire a $110{ }^{\circ} \mathrm{C}$.

A la vez se utilizó como agente encapsulante maltodextrina con $10 \mathrm{DE}$ (dextrosa equivalente) por tener pro- piedades de baja viscosidad a altas concentraciones de sólidos, que permiten la formación de libre flujo sin enmascarar el sabor original (García et al., 2004); así mismo, Bakowska-Barczaka y Kolodziejczykb (2011), al evaluar el contenido de compuestos bioactivos en la grosella negra de un conjunto de maltodextrinas con diferentes DE $(11,18$ y 21) encontró un mayor rendimiento y una mejor protección para los compuestos polifenólicos durante 12 meses a 8 y $25{ }^{\circ} \mathrm{C}$ de almacenamiento. Herazo (2013), en el microencapsulado de cáscara de berenjena, obtuvo valores entre 101,8 $\mu \mathrm{mol}$ trolox/g y 103,1 $\mu \mathrm{mol}$ trolox/g a $170{ }^{\circ} \mathrm{C}$ y $180{ }^{\circ} \mathrm{C}$ respectivamente.

\section{Antocianina monoméricas y capacidad an- tioxidante de la salchicha tipo Frankfurt}

En la Tabla 3, se reporta contenido de antocianina monomérica y capacidad antioxidante en salchichas tipo Frankfurt después de la operación de escaldado en las diferentes sustituciones.

Tabla 3. Contenido de antocianina monomérica y capacidad antioxidante en las diferentes sustituciones de salchichas tipo Frankfurt.

\begin{tabular}{ccccccc}
\hline \hline & \multicolumn{3}{c}{$\begin{array}{c}\text { Antocianina mo- } \\
\text { noméricas* } \mathbf{~ m g / 1 0 0 ~ g}\end{array}$} & \multicolumn{2}{c}{ TEAC $\boldsymbol{\mu m o l} / \mathbf{g}$} \\
\cline { 2 - 8 } & $\bar{X}$ & & $\sigma_{x}$ & $\bar{X}$ & & $\sigma_{x}$ \\
\hline \hline Testigo & $0,00^{\mathrm{d}}$ & \pm & 0,00 & $9,25^{\mathrm{b}}$ & \pm & 0,08 \\
\hline \hline $\mathrm{S} 1(100 \%)$ & $0,55^{\mathrm{a}}$ & \pm & 0,01 & $19,23^{\mathrm{a}}$ & \pm & 0,46 \\
\hline \hline $\mathrm{S} 2(75 \%)$ & $0,25^{\mathrm{b}}$ & \pm & 0,02 & $20,30^{\mathrm{a}}$ & \pm & 0,33 \\
\hline \hline $\mathrm{S} 3(50 \%)$ & $0,09^{\mathrm{c}}$ & \pm & 0,02 & $18,49^{\mathrm{a}}$ & \pm & 1,05 \\
\hline \hline
\end{tabular}

Nota: *mg de pelargonidina-3-glucosido/100 g de salchicha tipo Frankfurt, valores con diferentes letras ( $a, b$ y c) dentro de la columna denotan significancia, para $\mathrm{n}=3$

Las diferentes muestras de salchichas tipo Frankfurt a las diferentes sustituciones de nitritos por extracto antocianico atomizado presentan diferente contenido de antocianos; así mismo, estas se degradan por el efecto de la operación de escaldado debido a que el producto es sometido a una temperatura de $75^{\circ} \mathrm{C}$ por 20 minutos. Al respecto Zapata (2014), señala que las antocianinas sufren una degradación cinética de primer orden por encima de los $60^{\circ} \mathrm{C}$, observándose después del escaldado una coloración parda.

Por otro lado, se evidencia que no existe diferencia estadística $(p>0,05)$ en la capacidad antioxidante en las diferentes sustituciones (50\%, $75 \%$ y $100 \%$ ) de las salchichas tipo Frankfurt. También, la muestra testigo presenta un menor contenido de capacidad antioxidante con respecto a las diferentes formulaciones; este resultado es atribuido a la presencia de antioxidantes endógenos como los péptidos (carnosina), ácido úrico, poliaminas, ascorbato, enzimas y antioxidantes exógenos (polifosfato y especias) (Sacchetti et al., 2008). Al ser la formulación la misma para todas las muestras, dan- 
do mayor capacidad antioxidante la adición de extracto atomizado en las sustituciones, principalmente por la captación de radicales libres, siendo un buen indicativo para predecir la estabilidad oxidativa.

Isaza et al. (2012), emplearon carne de res y pasta de pollo con adición de extracto de cereza cuyos valores de capacidad antioxidante medidos por el radical DPPH• fueron de 0.095 a $1.117 \mu$ mol equivalente trolox/g de salchicha, comparando con los resultados de 9,25 a $20,30 \mu \mathrm{mol} / \mathrm{g}$ de salchicha obtenidos en laboratorio, estos difieren por la metodología empleada en la extracción de la parte hidrofílica de la salchicha.

Ozgen et al. (2006), mencionado por Viviane et, al. (2015), describe que cuando los antioxidantes son añadidos a los alimentos, como los productos cárnicos, es- tos se convierte en un sistema complejo y, por lo tanto, son susceptibles de someterse a una serie de interacciones y a diferentes mecanismos antioxidantes, tales como la transferencia de electrones, la transferencia de hidrógeno y de quelación de metales; este hecho podría haber sido una de las causas a los resultados observados, en el presente estudio, sobre la cuantificación de la capacidad antioxidante.

\section{Color de las salchichas tipo Frankfurt antes de escaldado y después de escaldado.}

La Tabla 4, reporta el color instrumental $\left(L^{*}, a^{*}\right.$ y $\left.b^{*}\right)$ en las diferentes sustituciones (50\%, $75 \%$ y $100 \%$ ) de nitrito de sodio por el atomizado antocianico de mastuerzo, incluido el tratamiento testigo bajo dos condi-

Tabla 4. Valores de luminosidad $(L *)$ y parámetros de cromaticidad $(a *$ y $b *)$ de las salchichas tipo Frankfurt

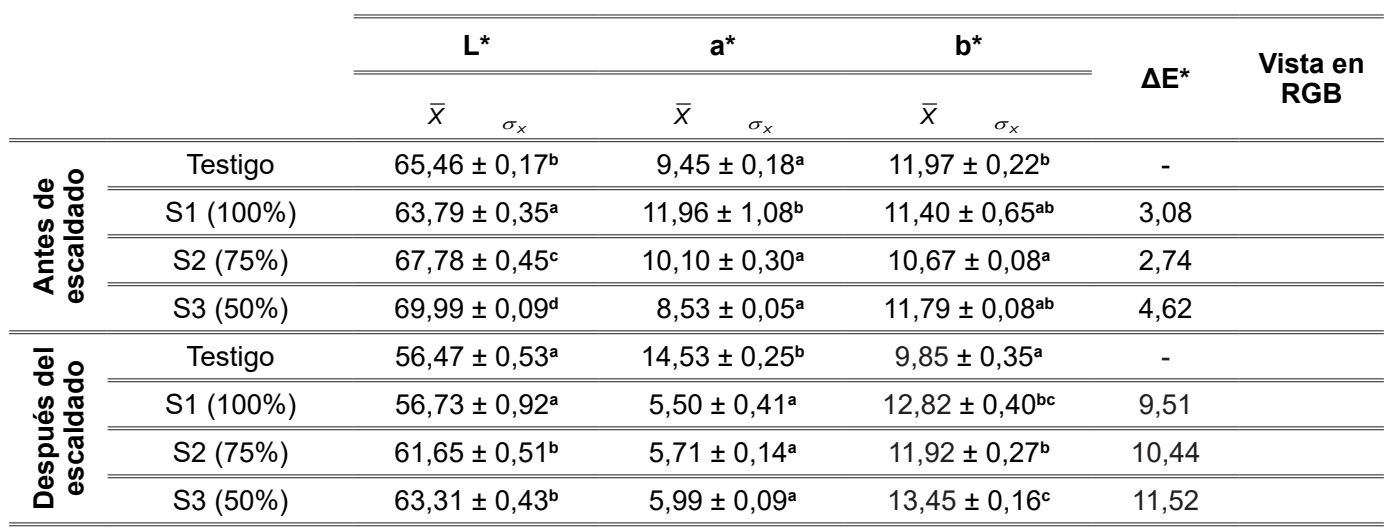

Nota: RVA son las siglas de los colores primarios (Rojo verde y azul). Letras diferentes en las columnas (a, b, c y d) expresan diferencia significativa $(p<0,05)$, para $\mathrm{n}=3$.

ciones: antes y después del escaldado de las salchichas tipo Frankfurt.

Los resultados de los tres parámetros de color (L*, a* $\left.\mathrm{y}^{*}\right)$ para las muestras crudas y escaldada muestran diferencia estadística $(p<0,05)$, se evidencia que la operación de escaldado afecta el color de las salchichas tipo Frankfurt. Al respecto Fennema (2000), señala que el calor en las salchichas permiten asegurar su vida útil, pero este influye en el parámetro a*, al estar relacionado con las antocianinas, y que estos pigmentos son sensibles a la exposición del calor, se evidencia esta variabilidad al pasar de 11,96 (antes de escaldado) a 5,50 (después de escaldado) probablemente por la formación de productos como el cumarín3,5-diglicósido y productos pardos que influyen en la coloración final del producto (Elbe y Schwartz, 2000).

De acuerdo a Sánchez-Zapata et al. (2010), señalado por Selani et al. (2016), establecen que las alteraciones causadas por el proceso de cocción (reacción de Maillard, desnaturalización de las proteínas, desnaturalización de grasa y pérdida de agua) podrían enmascarar algunos cambios del color no deseables inducidas por la formulación; a ello también se le atribuye la variación del color de las salchichas después de la cocción y no solamente a la pérdida del color por efecto al pigmento antocianico. Por otro lado, Ligianne et al. (2011) en la evaluación del extracto de uva sobre la carne de pollo ha encontrado diferencias significativas $(p<0,05)$ en los valores medios de b* (amarillo intensidad/azul) frente al tratamiento control y que esta variabilidad tiene relación directa con el extracto usado en la formulación.

Comparando la disminución de nitrito de sodio en la elaboración de salchichas Frankfurt adicionadas con extracto oleoso de residuos de chontaduro (Bactris Gasipaes), provocó un incremento en el valor de la luminancia $L * 75,54$ a 78,95 mientras que valor de croma a* disminuyó de 5,73 a 4,62 y el valor de croma b* se incrementó de 8,39 a 20,34 (Pinzón-Zárate, Hleap-Zapata y Ordóñez-Santos, 2015), estos resultados en la salchichas después del escaldado coinciden con lo reportado en este trabajo, encontrando similares valores de croma a* de 5,14 a 14,53 y b* de 9,85 a 13,45 en las diferentes sustituciones de nitrito a excepción de la luminancia $\mathrm{L}^{*}$, porque se ve influenciado por la materia prima y el extracto oleoso de chontaduro el cual contiene alto contenido de carotenoides (Jatunov et al., 2010 en Pinzón-Zárate, Hleap-Zapata y Ordóñez-Santos, 2015). 
El color instrumental, es un parámetro que no puede permitir evaluar la calidad de la salchicha tipo Frankfurt y de esta forma discriminar aquel producto (salchichas) que esté afectado groseramente por el efecto del calor. En la presente investigación, se omitió hacer las comparaciones con la muestra testigo, porque las salchichas con sustitución de nitrito de sodio por extracto antociánico después de haber sido escaldadas no alcanzaron visualmente el valor del croma a* (color rojo) muy ca- racterístico de las salchichas, la muestra testigo alcanzó un valor de 14,53 en el croma a* la más alta.

\section{Análisis microbiológico de las salchichas tipo Frankfurt}

La Tabla 5, reporta el análisis microbiológico de la salchicha tipo Frankfurt a las diferentes sustituciones (50\%, $75 \%$ y 1005) de nitrito de sodio por el atomizado antociánico del mastuerzo, incluida la muestra testigo, ello se

Tabla 5. Resultados de los análisis microbiológicos al día 1, 7 y 15 de las salchichas tipo Frankfurt

\begin{tabular}{|c|c|c|c|c|}
\hline \multirow{2}{*}{ Agente microbiano } & \multicolumn{4}{|c|}{ Día 1} \\
\hline & Testigo & n1 & n2 & n3 \\
\hline Aerobios mesófilos & $<10^{3}$ & $<10^{3}$ & $<10^{3}$ & $<10^{3}$ \\
\hline Escherichia coli & $<10$ ufc & $<10$ ufc & $<10$ ufc & $<10$ ufc \\
\hline Staphylococcus aureus & $<10$ ufc & $<10$ ufc & $<10$ ufc & $<10$ ufc \\
\hline Clostridium perfringens & $<10$ ufc & $<10$ ufc & $<10$ ufc & $<10$ ufc \\
\hline Salmonella sp. & Ausencia/25 g & Ausencia/25 g & Ausencia/25 g & Ausencia/25 g \\
\hline Listeria monocytogenes & Ausencia/25 g & Ausencia/25 g & Ausencia/25 g & Ausencia/25 g \\
\hline \multirow{2}{*}{ Agente microbiano } & \multicolumn{4}{|c|}{ Día 7} \\
\hline & Testigo & n1 & n2 & n3 \\
\hline Aerobios mesófilos & $<10^{3}$ & $<10^{3}$ & $<10^{3}$ & $2 \times 10^{3}$ \\
\hline Escherichia coli & $<10$ ufc & $<10$ ufc & $<10$ ufc & $<10$ ufc \\
\hline Staphylococcus aureus & $<10$ ufc & $<10$ ufc & $<10$ ufc & $<10$ ufc \\
\hline Clostridium perfringens & $<10$ ufc & $<10$ ufc & $<10$ ufc & $<10$ ufc \\
\hline Salmonella sp. & Ausencia/25 g & Ausencia/25 g & Ausencia/25 g & Ausencia/25 g \\
\hline Listeria monocytogenes & Ausencia/25 g & Ausencia/25 g & Ausencia/25 g & Ausencia/25 g \\
\hline \multirow{2}{*}{ Agente microbiano } & \multicolumn{4}{|c|}{ Día 15} \\
\hline & Testigo & n1 & n2 & n3 \\
\hline Aerobios mesófilos & $<10^{3}$ & $1 \times 10^{3}$ & $<10^{3}$ & $1 \times 10^{3}$ \\
\hline Escherichia coli & $<10$ ufc & $<10$ ufc & $<10$ ufc & $<10$ ufc \\
\hline Staphylococcus aureus & $<10$ ufc & $<10$ ufc & $<10$ ufc & $<10$ ufc \\
\hline Clostridium perfringens & $<10$ ufc & $<10$ ufc & $<10$ ufc & $<10$ ufc \\
\hline Salmonella sp. & Ausencia/25 g & Ausencia/25 g & Ausencia/25 g & Ausencia/25 g \\
\hline Listeria monocytogenes & Ausencia/25 g & Ausencia/25 g & Ausencia/25 g & Ausencia $/ 25 \mathrm{~g}$ \\
\hline
\end{tabular}

Nota: Valores obtenidos para para $\mathrm{n}=3$.

realizó al día 1, 7 y 15 el tiempo previsto de análisis está en función a la vida de anaquel de este tipo de embutidos. Los resultados del análisis microbiológico de la salchicha tipo Frankfurt en las diferentes formulaciones están por debajo de lo especificado para este tipo de embutidos y cumplen lo establecido por la NTS $\mathrm{N}^{\circ} 071$ (2002).

Al respecto Jai, Loessner y Golden (2005), señalan que las salchichas de diferente tipo, incluyendo las de Frankfurt, tienen como fuente de contaminación la formulación del resto de los ingredientes y especies. Muchas de estas especies, tienen elevadas cargas microbianas y la alteración de estos productos puede presentarse en tres formas: generación de limo, acidificación y aparición de un tono verdoso; el primero aparece en la cubierta, sobre todo de las salchichas Frankfurt, esto no se evidenció en las diferentes formulaciones, incluyendo el tratamiento testigo, debido a que se verificó la calidad de la materia prima, por lo que se afirma que es reflejo de los ingredientes.

Finalmente, podemos atribuir el efecto antimicrobiano que podría estar ejerciendo el pigmento antociánico en la elaboración de salchichas, debido a que las salchichas tipo Frankfurt, en las diferentes sustituciones, también permiten garantizar la calidad microbiológica de este producto. Los compuestos fenólicos y antocianos son 
responsables de la capacidad antioxidante y antimicrobiana (Zheng et al., 2011).

\section{Análisis sensorial de las salchichas tipo Frankfurt por escala hedónica}

En la Tabla 6, se muestra los resultados obtenidos de la evaluación sensorial con respecto a los atributos de color, olor, sabor y textura.

Tabla 6. Rangos de Kruskal Wallis para la evaluación de color, olor, sabor y textura.

\begin{tabular}{ccccc}
\hline \hline ATRIBUTO & SUSTITUCIÓN & $\mathbf{N}$ & $\begin{array}{c}\text { Sig. } \\
\text { asintotica }\end{array}$ & $\begin{array}{c}\text { Rango } \\
\text { promedio }\end{array}$ \\
\hline \hline \multirow{3}{*}{ COLOR } & $737(50 \%)$ & 32 & & $58,81^{\mathrm{a}}$ \\
& $835(75 \%)$ & 32 & 0,009 & $48,50^{\mathrm{b}}$ \\
& $733(100 \%)$ & 32 & & $38,19^{\mathrm{c}}$ \\
\hline \hline \multirow{3}{*}{ OLOR } & $737(50 \%)$ & 32 & & $50,31^{\mathrm{a}}$ \\
& $835(75 \%)$ & 32 & 0,232 & $53,05^{\mathrm{a}}$ \\
& $733(100 \%)$ & 32 & & $42,14^{\mathrm{a}}$ \\
\hline \hline \multirow{3}{*}{ SABOR } & $737(50 \%)$ & 32 & & $57,00^{\mathrm{a}}$ \\
& $835(75 \%)$ & 32 & 0,030 & $48,94^{\mathrm{b}}$ \\
& $733(100 \%)$ & 32 & & $39,56^{\mathrm{c}}$ \\
\hline \hline \multirow{3}{*}{ TEXTURA } & $737(50 \%)$ & 32 & & $52,88^{\mathrm{a}}$ \\
& $835(75 \%)$ & 32 & 0,454 & $48,09^{\mathrm{a}}$ \\
\hline \hline
\end{tabular}

Nota: Valores con diferentes letras (a, b y c) dentro de la columna denotan significancia.

Con respecto a los atributos de color y sabor, se evidencia diferencia estadística $(p<0.05)$ en las diferentes sustituciones (50\%, $75 \%$ y $100 \%$ ) de nitrito de sodio por el atomizado antocianico de mastuerzo, presentando los mejores atributos la formulación con el $50 \%$ de sustitución. Con respecto a los atributos olor y textura, se evidencia que no existe diferencia estadística $(p>0,05)$ todas las muestras con las diferentes sustituciones tienen la misma aceptabilidad.

Selani (2016), señala que el atributo color en productos cárnicos juega un papel decisivo en la evaluación sensorial y que de ella depende su aceptabilidad en productos cárnicos.

Ligianne et al, (2011), los atributos de cambio de color y olor a rancio en el análisis de carne de pollo cocinada con la adición de orujo de uva fueron significativamente diferentes a los 15 días de almacenamiento, lo que indica que puede haber un ligero deterioro de los productos que se perciben por los panelistas, en la investigación no se evidencia la diferencia de olor y que la variabilidad de la tonalidad del color en las salchichas tipo Frankfurt se debe al extracto atomizado de mastuerzo.

\section{Conclusiones}

Los resultados de este estudio, demostraron que el extracto antociánico atomizado con $9 \%$ de maltodextrina y secado por atomización a $110{ }^{\circ} \mathrm{C}$ pueden sustituir el uso de nitrito de sodio en un 50\% en la formulación de salchichas tipo tipo Frankfurt; así mismo, el producto elaborado presenta $0,09 \mathrm{mg}$ pgd-3-glu/ $100 \mathrm{~g}$ de antocianinas y 18,49 $\mu \mathrm{mol}$ Trolox/g de capacidad antioxidante, lo que lo hace factible para su uso en la industria de embutidos.

\section{Referencias bibliográficas}

Badui, D. S. (2006). Química de los alimentos. México: Pearson Educación. p. 417-420.

Bakowska-Barczaka, A., y Kolodziejczykb, P. (2011). Black currant polyphenols: Their storage stability and microencapsulation. Industrial Crops and Products. Vol. (34). p.1301 - 1309.

CODEX STAN 192. (1995). Norma general del Codex para los Aditivos Alimentarios. Recuperado de http: / / www.administracion.usmp.edu.pe/ institutoconsumo/ wp-content/uploads/2013/08/ Aditivos-Alimentarios-CODEX.pdf

Ligianne Din Shirahigue, Carmen J. Contreras-Castillo, Miriam Mabel Selani, Ana Paula Nadai, Gerson Barreto Mourão, Cláudio Rosa Gallo. (2011). Winery grape-residue extract: Effects on quality and sensory attributes of cooked chicken meat. Food Science and Biotechnology, Vol. 20, Number 5, Page 1257

Lara, E.; Martín, O.; Osorio, P.; Barrera, L.; Sánchez, J. y Bautista, S. (2014) Actividad antioxidante composición nutrimental y funcional de flores comestibles de dalia, Revista Chapingo Serie Horticultura; 20(1): 101-116.

Elbe, J., y Schwartz, S. (2000). Colorantes. España. Editorial Acribia.

Fennema, O. (2000). Introducción a la química de los alimentos. España. Editorial Acribia.

García, G., González, M., Ochoa, M., y Medrano, H. (2004). Microencapsulación del jugo de cebada verde mediante secado por aspersión. Revista Ciencia y Tecnología Alimentaria. Vol. (4). p. 262-266.

Garzón, G., y Wrolstad, R. (2009). Major anthocyanins and antioxidant activity of Nasturtium flowers (Tropaeolum majus). Food Chemistry. Vol. (114). p. 44 - 49.

Giusti, M., y Wrolstad, R. (2001). Characterization and Measurement of Anthocyanins by UV-Visible Spectroscopy. ResearchGate. USA. p. 1 - 14. 
Herazo, I. (2013). Obtención y estabilización de antocianinas de berenjena (Solanun melongena L.) mediante microencapsulación y su evaluación como compuesto funcional en la industria alimentaria. (Tesis de maestría). Colombia. p. 51-55.

Isaza, Y., Gil, J., López, J., Ochoa, O., y Restrepo, D. (2012). Capacidad antioxidante, a los 10 días de almacenamiento, de sistemas modelo de salchicha tipo Frankfurt adicionadas con extracto de cereza (Prunusavium L.) Rev. Fac. Ing. UCV. Venezuela. Vol. (27).

Jai, J.; Loessner, M. y Golden, D. (2005). Microbiología moderna de los alimentos. Quinta edición. Editorial Acribia. Zaragoza, España.

Kim, D., Lee, K., Lee, H., y Lee, C. (2002). Vitamin $C$ equivalent antioxidant capacity (VCEAC) of phenolic phytochemicals. Journal of Agricultural and Food Chemistry. USA. p. 3713-3717.

Kim, D., Lee, K., Lee, H., y Lee, C. (2002). Vitamin $C$ equivalent antioxidant capacity (VCEAC) of phenolic phytochemicals. Journal of Agricultural and Food Chemistry. USA. p. 3713 - 3717.

Liria, D. M. (2007). Guía para la evaluación Sensorial de alimentos. Instituto de Investigación Nutricional [IIN]. Perú. recuperado de http://es.slideshare. net/ evytaguevara/gua-para-la-evaluacin-sensorial-de-alimentos

López, A. E. (2008). Producción de bebida alcohólica de alta calidad. (Tesis para optar el título). Universidad de las Américas Puebla. México. Recuperado de http://catarina.udlap.mx/u_dl_a/tales/documentos/lpro/lopez_a_e/capitulo1.pdf

Minsa/Digesa-v.01. (2002). Norma sanitaria que establece los criterios microbiológicos de calidad sanitaria e inocuidad para los alimentos y bebidas de consumo humano. NTS N071. Perú.

Moyer, R., Hummer, K., Finn, C., Frei, B., y Wrolstad, R. (2002). Anthocyanins, phenolics, and antioxidant capacity in diverse small fruits:Vaccinium, Rubus, and Ribes. J.Agric. Food Chem. USA.

Pinzón-Zárate, L., Hleap-Zapata, J., y Ordóñez-Santos, L. (2015). Análisis de los parámetros de color en salchichas Frankfurt adicionadas con extracto oleoso de residuos de Chontaduro. Colombia. Vol. (26).

Placas 3MTM PetrifilmTM. (2009). Guía de interpretación: para recuento de Enterobacterias. U.S.A.
Rodas, M. (2005). Determinación de la concentración de nitritos y nitratos en salchichas ofertada que se comercializa en los supermercados de la ciudad capital. Universidad de San Carlos de Guatemala. Guatemala.

Sacchetti, G., Cocci, E., Pinnavaia, G., Mastrocola, D. y Dalla, M. (2008). Influence of processing and storage on the antioxidant activity of apple derivatives. International Journal of Food Science \& Technology. p. $797-804$

Selani, M.; Shirado, G.; Margiotta, G.; Rasera, M.; Marabesi, A.;Piedade, S.; Contreras, C. y Canniatti, B. (2016). Pineapple by-product and canola oil as partial fat replacers in low-fat beef burger: Effects on oxidative stability, cholesterol content and fatty acid profile. Meat Science, Vol. 115, , Pag. 9-15. doi.org/10.1016/j. meatsci.2016.01.002

Solís, J. (2005). Tecnología de carnes. Manual de prácticas. Universidad Nacional del centro del Perú. p. 39-49

Viviane G. Packer, Priscilla S. Melo, Keityane B. Bergamaschi, Miriam M. Selani, Nilda D. M. Villanueva, Severino M. de Alencar and Carmen J. Contreras-Castillo. (2015). Chemical characterization, antioxidant activity and application of beetroot and guava residue extracts on the preservation of cooked chicken meat. Journal of Food Science and Technology, Vol. 52, Number 11, Pag 7409-7416 DOI: 10.1007/ s13197-015-1854-8

Youngson, R. (2004). ¿Qué son los radicales libres? En: Antioxidantes y radicales Libres. Acribia. España.

Zapata, L., M. (2014). Obtención de extracto de antocianinas a partir de arándanos para ser utilizado como antioxidante y colorante en la industria alimentaria. (Tesis doctoral). Universidad Politécnica de Valencia. España.

Zheng, N., Z. Wang, F. Chen y J. Lin. (2011). Evaluation to the antioxidant activity of total flavonoids extract from Syzygium jambos seeds and optimization by response surface methodology. Journal Pharm Pharmacol. 21, 2411-2419. 